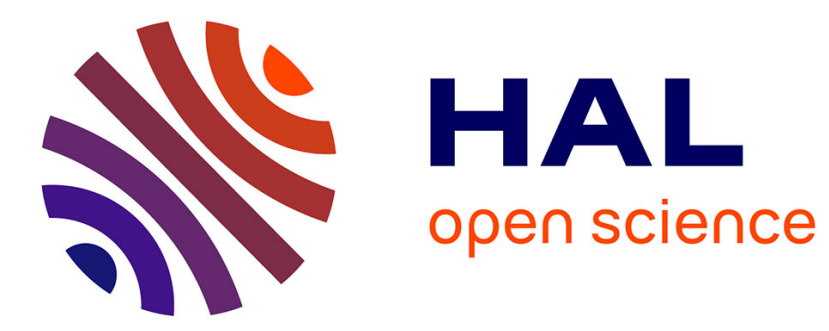

\title{
Les impacts de la RSE sur les systèmes de contrôle
}

Essid Moez, Nicolas Berland

\section{To cite this version:}

Essid Moez, Nicolas Berland. Les impacts de la RSE sur les systèmes de contrôle. Comptabilité Contrôle - Audit, 2011, 17 (2), pp.59-88. halshs-00637633

\section{HAL Id: halshs-00637633 https://shs.hal.science/halshs-00637633}

Submitted on 7 Nov 2011

HAL is a multi-disciplinary open access archive for the deposit and dissemination of scientific research documents, whether they are published or not. The documents may come from teaching and research institutions in France or abroad, or from public or private research centers.
L'archive ouverte pluridisciplinaire HAL, est destinée au dépôt et à la diffusion de documents scientifiques de niveau recherche, publiés ou non, émanant des établissements d'enseignement et de recherche français ou étrangers, des laboratoires publics ou privés. 


\title{
Les impacts de la RSE sur les systèmes de contrôle Consequences of CSR on
}

\section{management control system}

\author{
Moez Essid* et Nicolas BerLand **
}

\section{Résumé}

Cet article étudie les impacts de la Responsabilité Sociétale de l'Entreprise (RSE) sur le système de contrôle mis en place par une entreprise du secteur énergétique. Ces impacts sont analysés grâce au cadre théorique des leviers de contrôle proposé par Simons. La RSE a provoqué une surcharge cognitive des managers, ceux-ci étant soumis à un flot plus important d'informations. L'article s'intéresse alors aux conséquences de cette surcharge cognitive sur le design du système de contrôle mis en place et en tire quatre propositions sur 1/ la nature inappropriée du contrôle de la RSE comme candidat à un contrôle interactif, 2/ la substituabilité du contrôle diagnostique au contrôle interactif pour faire face à la surcharge cognitive, 3/ la spécialisation hiérarchique des informations nécessitant une discussion interactive et 4/ la focalisation de la discussion interactive sur des indicateurs communs à plusieurs niveaux hiérarchiques.

Mots ClÉS. - SYSTEMES DE CONTROLE RSE - CONTROLE INTERACTIF DIAGNOSTIQUE - SURCHARGE COGNITIVE.

\begin{abstract}
This paper is concerned with consequences of Corporate Social Responsibility (CSR) on management control design. These consequences are analysed through the model of levers of control developed by Robert Simon. CSR has increased the level of information overload due to the proliferation of data. The paper analyses the consequences on management control systems (MCS) of this information overload. It makes four proposals related to 1/the inadequacy of CSR management control system as an interactive system, 2/ the substitutability of the diagnostic control to interactive control to face the information overload, $3 /$ the specialisation of measures on each organisational level to implement interactivity and 4/ the focus for interactive discussion on information commonly used by different organisational levels.
\end{abstract}

KEYWORDS. - MANAGEMENT CONTROL
SYSTEMS - CSR - INTERACTIVE / DIAGNOSTIC
CONTROL - INFORMATION OVERLOAD

\footnotetext{
* Enseignant Chercheur, Institut Supérieur de Gestion de Paris

** Professeur à l'Université Paris Dauphine
} 
Correspondance : Moez ESSID

Institut Supérieur de Gestion de Paris GRIISG

147, Avenue Victor Hugo, 75116 Paris moez.essid@isg.fr
Nicolas BERLAND

Université Paris-Dauphine, DRM

Place du maréchal de Lattre de Tassigny

75775 Paris Cedex 16

nicolas.berland@dauphine.fr

\section{Introduction}

En élargissant la notion de performance, la Responsabilité Sociétale des Entreprises ${ }^{1}$ (RSE) offre l'opportunité de revisiter le fonctionnement des systèmes de contrôle. Néanmoins très peu de recherches ont étudié les systèmes de contrôle de la RSE (Quairel, 2006). La plupart des études en comptabilité-contrôle ont, en effet, porté sur les pratiques de communication extra financière (Oxibar, 2003 ; Damak Ayadi, 2006). Pourtant, la RSE contribue à mettre sous tension les systèmes de contrôle et fournit une opportunité pour mieux comprendre leur fonctionnement dans un contexte partiellement nouveau pour les acteurs qui vivent ces changements. Certaines entreprises mettent alors en œuvre des systèmes de contrôle de la RSE fonctionnant de façon intégrée avec les systèmes plus traditionnels.

Par extension à la définition initiale de Simons (1987a) des systèmes de contrôle, nous définissons les systèmes de contrôle de la RSE comme l'ensemble des procédures et systèmes formalisés, fondés sur l'information financière et extra financière, environnementale et sociétale, que les managers utilisent pour maintenir ou modifier certaines configurations des activités de l'organisation cherchant à améliorer la performance globale de l'entreprise.

Les systèmes de contrôle de la RSE s'intéressent ainsi à la performance sociétale, qui est désormais considérée comme partie intégrante de la performance organisationnelle, c'est-à-dire une performance prenant en compte un nombre accru de dimensions (Richard et al., 2009) au risque de surcharger l'attention des managers. Néanmoins, ces systèmes de contrôle sont encore en phase de construction. L'objectif de cette recherche est donc d'explorer ce champ relativement peu connu. Plus particulièrement, nous cherchons à comprendre quelles sont les conséquences de la mise en place d'une politique RSE sur les systèmes de contrôle?

Pour répondre à cette question, nous utiliserons le cadre théorique des leviers de contrôle de Simons (1995). Ce dernier permet d'analyser le design d'un système de contrôle au travers de quatre leviers, dont deux d'entre eux (les systèmes diagnostiques et interactifs) permettent plus spécifiquement d'analyser les systèmes de suivi et de reporting selon que les managers les utilisent pour surveiller la mise en place de la stratégie ou pour mieux comprendre les leviers stratégiques sur lesquels ils s'appuient.

La RSE est source d'incertitudes stratégiques pour les entreprises car elle redéfinit les marchés et les pratiques. Les multiples niveaux de performance qu'elle introduit compliquent la déclinaison de la stratégie et mettent les managers en situation de devoir apprendre à manier ces différents leviers d'amélioration de la performance. Compte tenu de la faiblesse des connaissances en ce domaine, nous avons opté pour une recherche par étude de cas. Celle-ci s'appuie sur une étude menée au sein d'une entreprise française du secteur énergétique pendant quatre ans.

Dans la première partie de cet article, nous rappellerons les enjeux théoriques associés aux systèmes de contrôle RSE et montrerons comment le cadre théorique développé par Simons (1995) peut aider à analyser les pratiques observées. Cette partie justifie également le cas étudié et décrit la méthodologie mise en œuvre. La deuxième partie de cet article présente l'étude de cas et le système de 
contrôle RSE mis en place. Enfin, la troisième partie discute des résultats empiriques et théoriques que nous tirons de notre étude de cas.

\section{Les systèmes de contrôle et la RSE}

Nous verrons d'abord comment les recherches sur les systèmes de contrôle ont abordé la RSE. Puis, dans un deuxième temps, nous montrerons comment le cadre théorique de Simons peut être mis à profit pour étudier une pratique de contrôle de la RSE. Enfin, nous présenterons l'entreprise étudiée, ainsi que la méthodologie employée.

\subsection{Systèmes de contrôle et RSE}

Les recherches traitant du contrôle de la RSE sont apparues il y a une décennie. Cette littérature a cherché initialement à identifier les outils de contrôle de la performance globale mis en place dans les entreprises. Le tableau 1 ci-dessous classe ces principaux outils selon deux axes (nature de la performance RSE mesurée : environnementale, sociale et globale, et niveaux d'application dans le processus de contrôle : planification, pilotage et audit et reporting).

Néanmoins, ces pratiques de contrôle sont fragmentées et partagées entre plusieurs directions de l'entreprise. Ce constat fait conclure à Germain et Gates $(2007, \mathrm{p} .11)$ que «le pilotage stratégique de la RSE est une pratique peu répandue dans les entreprises. Ce constat plaide en faveur de l'idée selon laquelle la problématique de la RSE demeure aujourd'hui confinée à des objectifs de communication externe ».

\section{Tableau 1}

Synthèse des principaux outils de pilotage de la RSE

(Adaptée de Capron et Quairel -Lanoizelée, 2004)

\begin{tabular}{|c|c|c|c|c|}
\hline & \multicolumn{3}{|c|}{ (Adaptee de Capron et Quairel -Lanoizelée, 2004) } \\
\hline & & \multicolumn{3}{|c|}{ Niveaux d'application } \\
\hline & & Planification & Pilotage & Audit et Reporting \\
\hline \multirow{3}{*}{$\begin{array}{l}\stackrel{D}{\Xi} \\
\frac{\mathrm{E}}{\mathrm{N}}\end{array}$} & Environnementale & $\begin{array}{c}\text { EMAS (Eco Management } \\
\text { and Audit Scheme) } \\
\text { ISO } 14000 \\
\text { Eco-conception }\end{array}$ & & $\begin{array}{c}\text { Bilan } \\
\text { environnemental } \\
\text { EMAS }\end{array}$ \\
\hline & Sociale & $\begin{array}{c}\text { OHSAS } 18000( \\
\text { Occupational Health and } \\
\text { Safety Assessment Series) } \\
\text { Norme SA (Social } \\
\text { Accountability) } 8000 \\
\end{array}$ & & Bilan social \\
\hline & Globale & $\begin{array}{l}\text { Sustainability Balanced } \\
\text { Scorecard } \\
\text { ISO } 26000 \\
\text { Navigateur Skandia AFS } \\
\text { (Assurance \&. Financial } \\
\text { Services) } \\
\text { AA (AccountAbility) } 1000\end{array}$ & $\begin{array}{c}\text { Sustainability Balanced } \\
\text { Scorecard } \\
\text { SD }(\text { Sustainable } \\
\text { Development }) 21000 \\
\text { Navigateur Skandia AFS }\end{array}$ & $\begin{array}{c}\text { GRI (Global } \\
\text { Reporting Initiative }) \\
\text { Bilan sociétal } \\
\text { AA } 1000 \\
\text { Comptabilité } \\
\text { sociétale }\end{array}$ \\
\hline
\end{tabular}

Dans le cadre de ces études sur les pratiques managériales, Zingales et Hockerts (2003) ont identifié quelques exemples d'entreprises ayant intégré un axe RSE dans leurs tableaux de bord prospectifs. 
Hockerts (2001) et Bieker (2002) ont étudié les exemples de Novo Nordisk ou de Shell qui ont mis en place des modèles particuliers du Sustainability Balanced Scorecard. Mais, ces modèles particuliers de tableaux de bord prospectifs souffrent de leur incapacité à mesurer simultanément un triple résultat puisque la performance finale mesurée par ces modèles est toujours économique. D'ailleurs Capron et Quairel-Lanoizelée (2006, p.13) rappellent que le modèle théorique du Sustainability Balanced Scorecard «ne constitue pas un dispositif permettant d'évaluer et de piloter une performance globale mais il élargit le pilotage économique aux dimensions sociétales. Les limites mises en évidence par les études empiriques et relatives aux difficultés de mise en ouvre du BSC classique sont renforcées par l'introduction de dimensions supplémentaires; les pratiques restent très focalisées sur les indicateurs "faciles» à renseigner, plutôt monétaires; les aspects plus qualitatifs sont mal pris en compte (Bieker, 2002) et les liens de causalité ne sont presque jamais analysés (Ittner et Larcker, 2004)».

Au-delà du repérage des outils, les recherches académiques qui ont étudié les systèmes de contrôle de la RSE peuvent être classées en trois courants. Le premier d'entre eux s'intéresse à l'institutionnalisation des pratiques de contrôle de la RSE. Moquet et Pezet (2006) décrivent ainsi un processus de mise en œuvre de la RSE chez Lafarge. Berland et Loison (2008) analysent l'émergence $\mathrm{du}$ "Responsible Care" dans le groupe Rhodia depuis les années 1970. Loison et Pezet (2006) rappellent que des groupes, tels que Pechiney dans les années 1960, prenaient déjà en compte des considérations environnementales ou sociales.

Un deuxième courant de recherche s'intéresse aux relations entre les dimensions des systèmes de contrôle. Moquet (2008) montre que ces systèmes, chez Lafarge et Danone, excluent par nature les aspects financiers. Elle montre qu'il existe des conflits entre les systèmes de contrôle sociétaux et les systèmes de contrôle financiers du fait des deux logiques contradictoires qui animent chacun de ces deux systèmes. Ce conflit s'explique ainsi par une logique managériale schizophrène (création de valeur financière et évolution dynamique vers une compétitivité sociétale) et un gouvernement d'entreprise toujours fortement « orienté actionnaire ». De leur coté, Meyssonnier et Rasolofo-Distler (2008) ont observé des résultats inverses et une bonne intégration des différentes dimensions dans la RSE dans un seul système de contrôle. En effet, Meyssonnier et Rasolofo-Distler (2008) ont discuté des spécificités d'un système de contrôle mis en place dans une entreprise sociale pour l'habitat. Ils concluent que ce système, de par notamment l'activité spécifique de l'organisation étudiée, intègre concrètement les objectifs RSE au même titre que les objectifs financiers. Henri et Journeault (2010) ont étudié l'impact des systèmes «d'eco-control» sur la performance économique et environnementale. Ils s'inscrivent dans des courants de recherche ayant étudié cette relation (Preston et O’Bannon, 1997; Orlitzky et al., 2003; Melnyk et al., 2003 ), ainsi que les recherches sur la comptabilité de gestion environnementale (Ifac, 2005 ; Burnett et Hansen, 2008).

Un troisième courant s'est intéressé aux facteurs de contingence des modes de contrôle de la RSE (Marquet-Pondeville, 2003). L'impact de la stratégie des entreprises sur la manière dont celles-ci mettent en place des modes de contrôle a essentiellement retenu l'attention. Cette analyse est intéressante car elle complète finalement les études traditionnelles sur les relations stratégies-contrôle (Langfield-Smith, 1997).

Notre recherche vise à analyser plus spécifiquement les modalités de pilotage de la RSE et permet d'enrichir les réflexions sur les deux premiers axes de recherche développés ci-dessus. 


\subsection{Le cadre des leviers de contrôle de Simons comme grille d'analyse des systèmes de contrôle RSE}

Le cadre théorique des leviers de contrôle proposé par Simons $(1991,1994,1995)$ s'intéresse à la façon dont les systèmes de contrôle sont utilisés par les managers pour atteindre leurs objectifs, plutôt qu'à la nature des outils mis en œuvre. Il est donc pertinent avec l'objectif de notre travail.

Selon Simons (1995, p.175) quatre leviers de contrôle servent à «inspirer l'adhésion des individus aux buts de l'organisation; baliser le territoire d'expérimentation et de compétition, coordonner et piloter l'exécution des stratégies du moment; stimuler et guider la recherche de stratégies futures.». Les systèmes de croyances et de contraintes sont les deux premiers. Toutefois, ils ne nous intéressent pas directement dans le cadre de cette étude qui se focalise sur les deux autres leviers de contrôle ${ }^{2}$. En effet, deux autres leviers de contrôle recouvrent ce qui est traditionnellement couvert par les systèmes de contrôle. Ils servent à solliciter l'attention des acteurs de deux manières différentes (Simons, 1991, 1994, 1995) :

- Par exception avec un système de contrôle diagnostique. C'est la forme classique et cybernétique du contrôle. Pour Simons (1995, p. 60), « la quasi-totalité des écrits en contrôle de gestion se réfèrent à des systèmes de contrôle diagnostique ».

- De façon intensive avec le système de contrôle interactif. Il s'agit pour les acteurs d'interagir très fortement entre eux pour traiter des priorités stratégiques qui ont été définies a priori comme des facteurs clé pour l'entreprise.

Simons présente une grille de lecture théorique des outils de contrôle utilisés par les managers en situation de changement stratégique et d'incertitude. Elle permet d'analyser les systèmes de contrôle orientés vers le respect de la conformité de dimensions de la performance clairement institutionnalisés d'une part, et l'apprentissage de nouvelles stratégies et de nouvelles performances chemin faisant d'autre part. La mise en œuvre de nouvelles stratégies RSE, encore mal définies, devrait par conséquent avoir un impact sur les dimensions interactives et diagnostiques des systèmes de contrôle de la RSE mises en place ainsi que sur les systèmes de croyances et de contraintes.

Le cadre de Simons a suscité de larges débats dans la littérature. Le plus important d'entre eux concerne la nature du contrôle interactif (Osborn, 1998 ; Abernethy et Brownell, 1999; Marginson, 2002 ; Bisbe et Otley, 2004 ; Bruining et al., 2004 ; Dambrin et Loning, 2008). Mais plus spécifiquement, trois de ces débats semblent particulièrement concerner le contrôle de la RSE.

Le premier débat concerne le risque de surcharges cognitives (Schick et al., 1990) que le contrôle interactif fait peser sur les managers. Comme le montre Pizzini (2006), les managers semblent apprécier des informations détaillées pour pouvoir engager des débats en profondeur. Mais l'utilisation interactive du système de contrôle nécessite de la disponibilité intellectuelle de la part des managers. Pour permettre d'augmenter la volumétrie des données produites, ou pour rassembler les cadres dans des réunions favorisant le dialogue et l'interactivité, le système de contrôle doit donc être adapté. La question est alors de savoir comment le système de contrôle est modifié par des situations de surcharge cognitive. Selon Osborn, (1998), le système de contrôle semble devoir être semi formel afin de faire preuve de suffisamment de plasticité pour permettre une bonne prise en charge par les managers. Toutefois, et comme le montre Tuomela (2005, p.312) «le dialogue constructif (...) entraîne aussi des coûts non négligeables. En général, les managers ressentent une frustration et une perte de temps en assistant à toutes ces réunions alors qu'ils pensent qu'ils devraient faire ce que l'on attend d'eux qu'ils fassent ». Enfin, Simons (1991) lui-même a montré comment des organisations en phase de transition avec uniquement des systèmes interactifs, peuvent mettre des salariés dans des situations stressantes. Or, le contrôle de la RSE se matérialise par un volume d'information accrue qui peut être à 
la fois financier (coût des pratiques pour la protection de l'environnement par exemple) et non financier (taux de rejet de pollution, des incidents ou des actions entreprises). En conséquence, la RSE est un cas idéal pour étudier les comportements des systèmes de contrôle en cas de surcharge cognitive. Les informations utilisées par la RSE se caractérisent également par une incertitude plus forte que les informations financières, industrielles et commerciales (Lingle et Schiemann, 1996).

Le deuxième débat concerne le lien entre contrôle (interactif / diagnostique) et indicateurs non financiers. Le contrôle de la RSE nécessite une production importante d'indicateurs non financiers. Or, comme l'ont notamment montré Epstein et Manzoni (1997), les managers résistent parfois à la mise en place d'indicateurs non financiers car ces derniers augmentent la visibilité des actions qu'ils ont mises en œuvre. Les managers peuvent en effet se sentir menacés par l'existence d'indicateurs qui mettraient l'accent sur leurs domaines de responsabilité et limiteraient leurs possibilités de se protéger des questionnements et des examens minutieux (Vaivio, 1999). Cela est vrai notamment quand ces indicateurs font l'objet d'une utilisation interactive suscitant de nombreux débats et questionnements. L'introduction d'indicateurs non financiers risque également de rompre la structure des pouvoirs à l'intérieur d'une organisation (Vaivio, 2001). Tuomela (2005) montre qu'il existe une forme de résistance au changement, de la part de certains managers, pour l'adoption de systèmes de contrôle interactifs, fondés sur des informations non-financières détaillées. Cette forme de résistance risque d'être accrue dans le cas du contrôle de la RSE, du fait de l'incertitude portant sur les informations produites (risque d'être jugé de façon non équitable), mais aussi parce que les informations issues de la RSE sont fortement chargées de valeurs liées au respect des autres. Leur impact émotionnel risque donc d'être plus fort. La question est alors de savoir comment les managers réagissent et adaptent le système de contrôle dans le cas où davantage d'indicateurs non-financiers sont utilisés, ce qui est précisément le cas avec la RSE.

Enfin, le troisième débat concerne les interactions entre les leviers de contrôle (Widener, 2007). Des recherches ont mis en évidence des interactions entre le contrôle interactif et diagnostique qui définissent ensemble un «control mix » (Abernathy et Chua, 1996 ; Collier, 2005). La question est alors de savoir si ces systèmes sont substituables ou complémentaires. Simons (1994) a montré comment une relation dialectique peut se mettre en place là où des systèmes, d'abord utilisés de manière diagnostique, se transforment en système interactif en fonction de l'usage qu'en font les managers. Cette problématique est prégnante pour le contrôle de la RSE dans la mesure où une bonne partie de l'information sert à produire des reportings internes et externes, c'est-à-dire utilisée de manière diagnostique. Dès lors que les informations liées à la RSE sont aussi utilisées de manière interactive afin de faire face aux incertitudes stratégiques et managériales posées par la RSE, on ne peut ignorer ses interactions avec le système de contrôle diagnostique.

L'étude de cas que nous restituons ci-dessous permet d'analyser un système de contrôle cherchant à réduire les incertitudes stratégiques relatives aux problématiques de la RSE chez un groupe énergétique français.

\subsection{Présentation du cas et méthodologie}

Après avoir présenté l'entreprise étudiée, que nous nommerons Energetix, et justifié de son choix, nous exposerons notre démarche méthodologique. 


\subsubsection{PRESENTATION ET JUSTIFICATION THEORIQUE DU CHOIX DU CAS ENERGETIX}

Energetix est un groupe français né en 2001, spécialisé dans la production et l'acheminement d'énergie. Le groupe se compose aujourd'hui de 350 entités juridiques regroupées en quatre pôles. Présent dans un secteur industriel sensible en termes d'environnement et de sécurité, l'entreprise est particulièrement sous surveillance des groupes de pression externe. Les différentes parties prenantes interrogent sans cesse la pertinence des choix de l'entreprise, critiquent ses résultats, et souhaitent même parfois sa suppression.

Par ailleurs, la restructuration récente du groupe s'est accompagnée de l'arrivée d'un nouveau PDG qui a cherché à marquer les esprits par un degré d'ouverture aux parties prenantes, au moins en apparence, plus grand. Ce choix a constitué une rupture stratégique forte pour le groupe, et a provoqué un bouleversement culturel qui s'est traduit jusque dans les pratiques de gestion.

Ces bouleversements managériaux se sont matérialisés par un nouveau système managérial: l'Energetix Way. Ce dernier se présente comme un système de pilotage où sont intégrées sur le même plan les différentes dimensions de la performance RSE (économique, financière, environnement, sécurité, sociale et sociétale). Ce système implique régulièrement et fortement les managers de différents niveaux hiérarchiques dans des réunions où sont passés en revue, afin d'être discutés, les différents éléments constituant la performance RSE de l'entreprise.

La naissance récente du groupe, son pari stratégique pour des orientations environnementales et sociétales, son système managérial intégré, ainsi que la nature même de ses activités, en font un terrain d'étude propice et riche en enseignement quant à la mise en œuvre des stratégies RSE et des outils de pilotage qui les accompagnent. En outre, le cas Energetix présente une situation permettant d'observer une intégration des dimensions fondamentales de la RSE à la stratégie. Les managers ont une nouvelle incertitude stratégique à gérer qui devrait se manifester dans leurs modes de pilotage.

\subsubsection{DEROULEMENT DE L'ETUDE EMPIRIQUE}

Nous nous sommes adressés en premier lieu à la direction du développement durable du groupe et qui, après plusieurs relances, a accepté notre projet d'étude. Le but de l'étude était de comprendre le fonctionnement d'un système de contrôle mis en place deux ans avant l'arrivée des chercheurs dans l'entreprise et dont ils avaient eu connaissance lors d'un colloque professionnel. Les chercheurs ont eu une situation d'observateurs et ne se sont jamais trouvés en situation d'intervention dans l'entreprise. Seul un compte-rendu d'observation a été effectué à l'issue de deux périodes, après avoir examiné le système d'un point de vue macro au niveau de la direction et après avoir pu assister aux modalités de fonctionnement de ce système dans l'une des BU (Business Unit). Ces comptes-rendus ont fait l'objet de discussions avec les managers de l'entreprise et ont permis d'enrichir notre compréhension des enjeux. Les observations portant sur des processus de gestion et non la teneur des actions RSE, les débats étaient relativement dépassionnés. En effet, les cadres de l'entreprise affectaient une très grande modestie dans leurs pratiques qu'ils considéraient comme expérimentales.

Nous avons analysé la démarche «développement durable $»^{3}$ du groupe entre 2005 et 2008. En tout, nous avons rencontré et interviewé, à plusieurs reprises, quatorze acteurs intervenants dans la démarche «développement durable » (annexe 1):

- Au niveau groupe: deux personnes ont été initialement rencontrées (le directeur développement durable et son adjoint qui deviendra en cours d'étude le chef de la BU étudiée) 
afin d'avoir une description d'ensemble de la démarche : historique, organisation, principaux objectifs, principaux intervenants, etc.

- Au niveau Business Units : pour approfondir notre étude sur la mise en place de la démarche développement durable du groupe, nous avons eu l'opportunité d'examiner le processus au niveau d'une des BU, spécialisée dans le domaine de la chimie. Elle rassemble l'ensemble des activités chimique du groupe qui se matérialise en la conversion des matières premières et la transformation des concentrés miniers nécessaires à la production de l'énergie. En tout huit personnes ont été rencontrées au niveau de la direction générale de cette BU. Nous avons également assisté à trois réunions du comité de direction (Codir) de la BU, dédiées au suivi d'Energetix Way. Chacune de ses réunions avait duré une journée.

- Au niveau unités opérationnelles : la BU étudiée est composée de quatre usines de production implantées dans le sud de la France. Dans le cadre de notre étude, nous avons suivi la mise en place de la démarche dans ces usines en rencontrant à plusieurs reprises les quatre directeurs de ces établissements.

Par ailleurs, les entretiens conduits avec ces différentes personnes et notre présence lors des trois réunions du Codir ont permis d'accéder à une quantité importante de documents internes de la BU et de ses entités industrielles. Ces documents se sont avérés forts utiles pour notre recherche car ils ont rendu possible une autre vision, plus synthétique et plus factuelle, de l'ensemble de la démarche RSE du groupe. Ils nous ont également éclairé sur certains points restants mal définis à l'issue des entretiens, et ont permis de mettre au jour quelques différences d'utilisation des outils entre ce qui se fait réellement et ce qui est prescrit par les documents internes du groupe.

Les entretiens et des documents recueillis lors de l'étude longitudinale ont été par la suite analysés et traités à l'aide du logiciel de traitement des données qualitatives N'Vivo 2. Cette analyse thématique des données empiriques (Tesch, 1990 ; Miles et Huberman, 2003) s'est faite en plusieurs étapes :

- Codage des données en partant d'un ensemble de thèmes et de catégories issus à la fois de la revue de la littérature et des observations sur le terrain ;

- Condensation et réorganisation des données à la fin du codage en fonction des questions de recherches posées ;

- Interprétation des données.

\section{L'Energetix Way ou le pari d'un système de contrôle interactif de la RSE}

Pour décrire les pratiques d'Energetix, nous nous focaliserons sur la description des modes de contrôle interactif et diagnostique de la RSE utilisés et leurs finalités. Nous analyserons également l'articulation contrôle interactif/contrôle diagnostique des outils du système de pilotage RSE mis en place, ainsi que son évolution dans le temps.

\subsection{Un système de contrôle « intégré » pour piloter la RSE}

Le pilotage de la performance chez Energetix est un pilotage «global », intégrant simultanément les trois formes de performance organisationnelle : économique, sociale et environnementale. Il se base sur plusieurs outils (voir fig.1), que nous avons voulu organiser et présenter selon les trois phases du processus de contrôle (Bouquin, 2008) : finalisation des objectifs, pilotage et post-évaluation. 
Figure 1

Le processus de pilotage de la performance globale chez Energetix

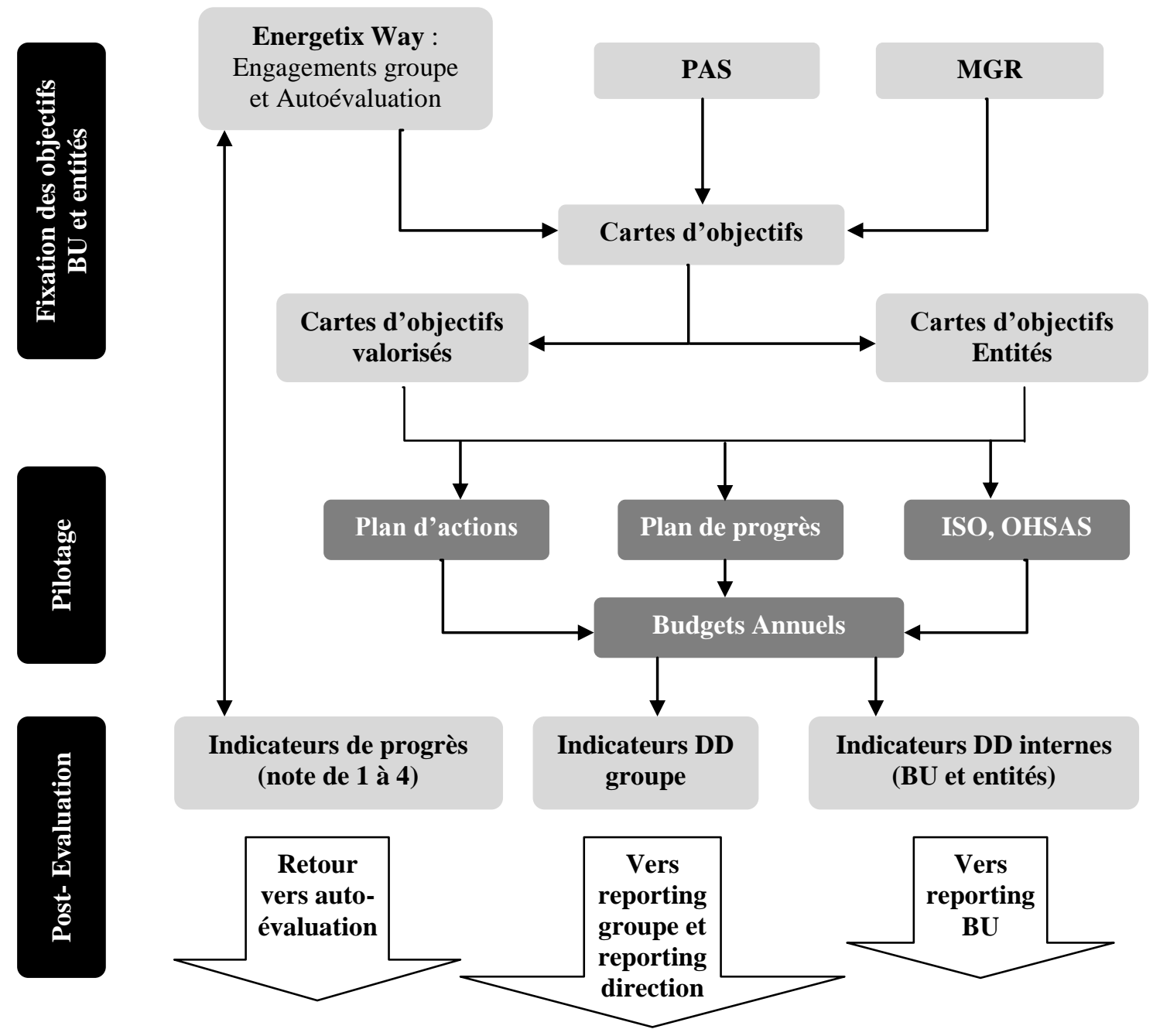

\subsubsection{LA FIXATION DES OBJECTIFS}

Sans être encore totalement maîtrisé, le processus de fixation d'objectifs RSE s'articule autour du système d'autoévaluation d'Energetix Way (voir fig. 1). Préalablement à la fixation des objectifs, des autoévaluations sont réalisées au niveau des unités opérationnelles. Elles mettent en évidence, selon une liste d'items préétablis, les points forts et les points faibles de chaque unité à partir d'un ensemble prédéfinis de critères d'amélioration issus des 10 axes stratégiques du groupe (annexe 2). Ceci permet de dégager les principales actions prioritaires qui alimenteront le plan d'action stratégique (PAS, qui représente le plan business du groupe, formé essentiellement d'objectifs commerciaux). Les différents objectifs sont par la suite hiérarchisés et intégrés dans des « cartes d'objectifs».

L'intérêt d'une telle triangulation est de permettre au groupe, et aux différentes unités opérationnelles, de construire des objectifs intégrant simultanément les différents aspects RSE dans un seul et même système de management. Cela se manifeste concrètement si on analyse la répartition des objectifs de la BU en 2005. On constate, au travers de cette analyse (fig.2), que les aspects sécuritésureté occupent une place équivalente à celle des objectifs économiques. 


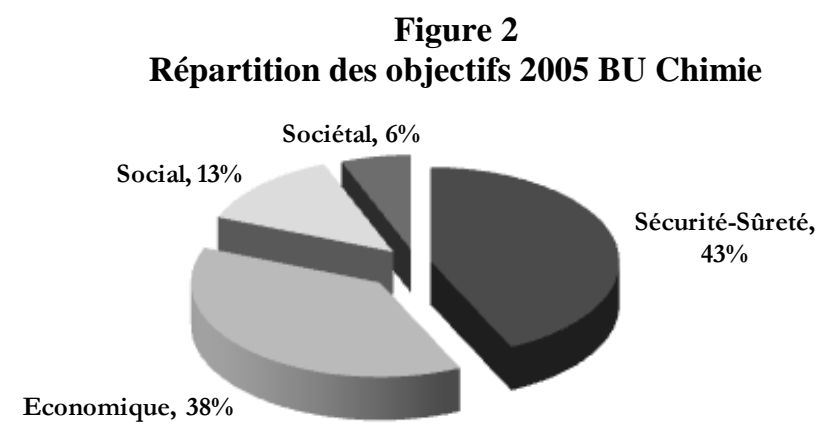

\subsubsection{LE PILOTAGE}

Le pilotage de la RSE se fait essentiellement au travers des plans d'action ou des plans de progrès. Ces derniers sont directement issus de l'autoévaluation et de l'ensemble des objectifs préétablis, puisqu'ils sont déclinés depuis les cartes d'objectifs.

"Un plan de progrès présente les objectifs de progrès continu de la BU et les principales actions de progrès mis en ouvre pour l'atteinte des objectifs. Il contient, comme la carte d'objectifs, les cibles et les impacts financiers pour l'année du budget... » Document Interne.

Ces plans font l'objet d'intégration au sein des budgets traditionnels et classiques des BU et des sites. Il y a donc la volonté de ne pas déconnecter les processus classiques de management des dimensions de la RSE. Le souci d'égalité entre les différentes dimensions de la RSE apparaît d'ailleurs clairement dans la répartition des plans de progrès de la $\mathrm{BU}$.

\section{Figure 3}

Répartition des plans de progrès 2005 BU Chimie

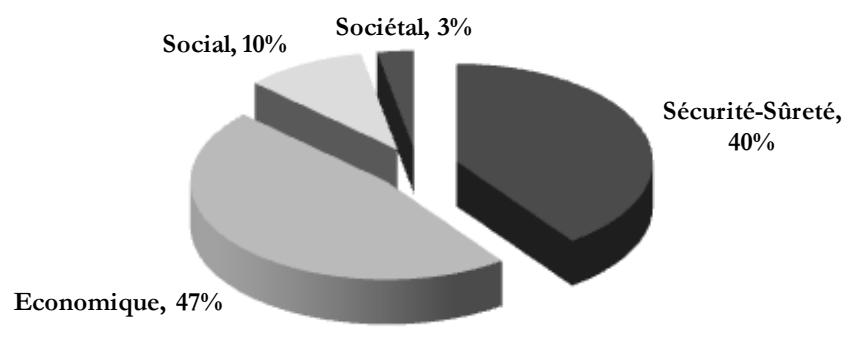

\subsubsection{LA POST-EVALUATION}

Enfin, la post-évaluation se base sur un ensemble large d'indicateurs de performance. Cet ensemble se compose d'indicateurs de progrès issus de l'autoévaluation (des notes de 1 à 4 pour les réalisations sur les critères d'amélioration) et d'indicateurs de performance provenant du suivi des plans d'action. Ces indicateurs servent en même temps au reporting groupe et au pilotage interne.

Les indicateurs de performance revêtent une importance considérable dans le management du groupe et de la BU. Toute action, projet ou réalisation, donne lieu rapidement à la production d'un nouvel ensemble d'indicateurs. Ceci s'explique probablement par une forte culture d'indicateurs au sein du groupe Energetix.

«En raison du grand nombre d'ingénieurs dans le groupe, nous croyons aux chiffres, peut-être trop car tout ne se résume pas à cela, mais nous avons mis en place des indicateurs et nous allons établir des objectifs chiffrés systématiques sur les différents indicateurs associés à des principes d'action et à des priorités que nous définissons. » PDG du groupe (document externe). 
Par ailleurs, l'Energetix Way étant un travail collaboratif et participatif, la responsabilité de la mesure et du suivi des indicateurs est déléguée à différents acteurs, des opérationnels ou des fonctionnels, s'occupant chacun d'un groupe d'indicateurs spécifiques.

"Sur l'établissement, il y a une personne chargée de constituer des groupes d'autoévaluations mettant des cadres et des agents de maitrise, en essayant de mettre des personnes concernées. Il peut y avoir des choses évaluées plusieurs fois. On partage ensuite le résultat en CODIR. On regarde les notes et on s'interroge de manière macro mais pas en reprenant un par un. On regarde ceux qui correspondent à des points sur lesquels on veut avancer. Après en cours d'année, on recense les items sur lesquels on a décidé de progresser et il y a des tableaux de bord et des plans d'actions. » Directeur d'un établissement industriel de la BU.

Ce mécanisme est le moyen trouvé par la direction pour faire participer le plus grand nombre possible de responsables dans ce système. L'idée est de s'assurer de l'adhésion d'une grande partie des salariés.

$\mathrm{Au}$ final, le système mis en place est assez classique. Il est toutefois aussi original parce qu'il traite de multiples sources de performance liées à la RSE. Nous allons maintenant l'analyser au travers des leviers interactifs et diagnostiques de Simons afin d'en présenter une approche plus dynamique.

\subsection{Les leviers de contrôle de la performance RSE}

Energetix a développé des systèmes interactifs et diagnostiques pour piloter la performance RSE aux niveaux opérationnels.

Le système de contrôle de la RSE mis en place avec Energetix Way, présente, dans les premiers temps de sa mise en place, les caractéristiques d'un système de contrôle interactif si l'on se réfère aux critères énoncés par Simons :

- Les outils de contrôle servent la démarche de progrès continu d'Energetix et implique une remise en cause de ses objectifs et actions. Ainsi, au travers de l'Energetix Way, le progrès de l'entreprise est visualisable par l'atteinte de niveau de maitrise croissante (ou de recul dans certains cas que nous avons eu à connaître) de chacun des critères du référentiel d'autoévaluation.

- Les instruments de contrôle impliquent intensément et régulièrement des opérationnels et des fonctionnels à différents niveaux afin d'inculquer le changement stratégique :

«L'autoévaluation par son rythme reconductible est un point positif, cela permet de se poser des questions mais il faut y inclure plus de personnes pour que ce ne soit pas un exercice en tour d'ivoire. Jusqu'ici ce n'est que le Codir, cette année, on a impliqué des ingénieurs, l'année prochaine ont impliquera des agents de maîtrise. » Directeur d'un établissement industriel

- Les critères d'amélioration d'Energetix Way sont en perpétuelle évolution et modification, puisqu'ils doivent être essentiellement issus de la discussion et de l'échange ${ }^{4}$.

- Une faible indexation de la rémunération sur les objectifs environnementaux ou sociaux est prévue par le système. Le système de rémunération reste essentiellement centré sur des dimensions financières et est géré plutôt par les processus Ressources Humaines, en dehors donc de l'Energetix Way.

- Le groupe utilise une forte communication interne, et un ensemble de programmes de formation sur la RSE, pour décliner et opérationnaliser les nouvelles valeurs.

- Seule la fréquence de l'examen des informations produites par l'Energetix Way peut faire douter de sa nature interactive. La discussion a lieu plusieurs fois par an et à différents niveaux de l'entreprise mais avec une fréquence finalement assez faible (tous les deux ou trois mois en 
moyenne). Le mécanisme observé n'est toutefois pas sans rappeler celui décrit chez Johnson et Johnson par Simons (1987b).

A côté de ce système interactif fonctionne également un système diagnostique composé de plusieurs outils. Le groupe a mis en place un système d'information (Star) et des tableaux de bord qui permettent la collecte décentralisée d'un grand nombre d'indicateurs non financiers liés à la RSE. De Star, sont sorties des informations standardisées, donc agrégeables qui servent de base au reporting de la direction générale et lui permettent de s'assurer de l'état de l'entreprise. A côté de Star fonctionnent des logiciels comptables et budgétaires plus traditionnels qui assurent le suivi des informations financières. S'ils sont consultés par les managers, ils ne constituent pas pour autant l'outil permettant les échanges et les réunions régulières comme cela peut être le cas avec Energetix Way.

\subsection{L'évolution de l'articulation contrôle diagnostique/contrôle interactif}

Le système a fortement évolué durant les quatre années où nous avons suivi le groupe. Ceci se matérialise par des changements dans l'utilisation du système par les managers qui :

- Négligent la discussion et l'échange recommandés par le système. Les réunions deviennent de plus en plus des passages obligés où les managers ne sont intéressés que par les quelques minutes où les informations qui les concernent sont discutées. L'échange se transforme alors en dialogue entre le chef et un seul manager subordonné à la fois.

- Passent de moins en moins de temps sur l'ensemble des critères d'amélioration, privilégiant ceux qui affectent directement la performance économique de leur entité. Cela ne signifie pas que la sécurité et l'environnement ou le traitement des dimensions sociales ne sont plus prises en compte mais elles sont traitées dans d'autres réunions qui ont lieu en amont du Codir dans les usines. La revue de performance dans ces autres niveaux se réalise peut-être, et même sans doute, via le référentiel Energetix Way puisque nous avons récupéré des traces écrites de ces échanges et des systèmes d'information. Formellement toutefois, les différentes étapes ont été découplées alors qu'elles devaient être simultanées dans le système tel qu'il avait été conçu initialement ${ }^{5}$.

- Ne s'intéressent aux dimensions non économiques, qu'en cas d'exception ou de défaillances importantes comme le montre le schéma ci-dessous. Les managers ont fait un travail de priorisation finalement assez conventionnel en réservant, de fait, les réunions de Codir aux dimensions économiques et financières, le reste étant traité plus localement, souvent dans les usines.

Figure 4

Répartition des objectifs 2007 BU Chimie

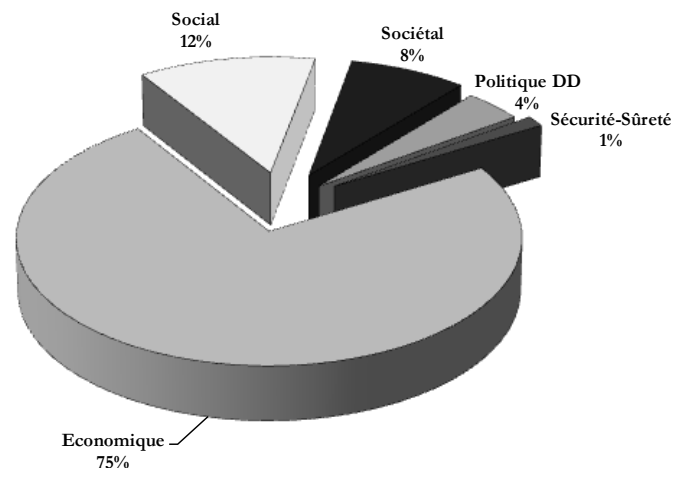

On constate, que bien qu'ayant gardé la même philosophie et les mêmes outils, une bonne partie du système a subi un glissement important pour passer d'une utilisation interactive dans les premières 
années pour l'ensemble des dimensions pertinentes de la performance à une utilisation diagnostique au bout de quelques temps pour des indicateurs autres qu'économiques. Les dimensions typiquement RSE ne font plus systématiquement partie d'un «agenda » récurent du Codir et la discussion est renvoyée vers d'autres instances afin de gagner du temps dans des réunions très chargées.

Les premières réunions s'étalaient sur huit heures. Au bout de quatre heures, seuls 25\% des items avaient fait l'objet d'une discussion (pour le coup très intense). Pris par le temps, le tempo s'accélérait alors et de nombreux items étaient laissés de côté, non par manque d'intérêt mais par manque de temps. Les managers avaient semble-t-il du mal à prioriser. Le travail de priorisation se faisait par défaut. Les réunions ont finis par être plus courtes mais en renvoyant certaines analyses à d'autres sessions. Relevés d'observation sur la tenue des réunions

La part des informations environnement-sécurité-social-sociétales discutées en Codir diminue et fait l'objet de discussions moins approfondies que les dimensions économiques. Ces informations sont toujours discutées mais dans des réunions ad hoc, sur les sites de production eux-mêmes. Il y a donc une sorte de découplage et de spécialisation des lieux de discussions des différents niveaux de performance.

Il ne reste plus du projet interactif initial qu'une discussion en Codir des performances principalement économiques et accessoirement RSE. Cela ne signifie pas pour autant que les managers se sont désintéressés des autres dimensions de la performance. Elles ont été traitées ailleurs d'une part, mais elles ont aussi été examinées sur la base d'un extrait du reporting «Star » d'autre part. En effet, un ensemble de 12 indicateurs normalisés (2 sécurité, 4 dosimétrie et 6 environnement), issus distinctement du système de reporting du groupe (qui correspond à plus d'une centaine d'indicateurs), et directement destinés à la direction générale, est devenu le centre d'intérêt récurrents et fréquents des managers. Autrement dit, un système diagnostique au début du processus est devenu de fait interactif au fil du temps.

Sous leur forme, ces indicateurs semblent être l'exemple type d'outils de contrôle diagnostique, qui ne sont remis en cause et étudiés qu'en cas d'exception. Mais l'importance accordée par la direction générale à ces indicateurs, et la difficulté des managers à « dompter » Energetix Way, a fait de sorte qu'une attention particulière est accordée à cet ensemble d'indicateurs lors des réunions auxquelles nous avons assistées.

Cette attention particulière montre que l'utilisation de ces indicateurs s'est transformée avec le temps pour passer de diagnostique à interactif. Pour autant, un temps conséquent était consacré à noter que ces indicateurs étaient de piètres informateurs de ce qui se passaient dans la BU. Malgré ces critiques, ces 12 indicateurs ont occupé les réunions.

\subsection{Des managers dépassés par le volume d'informations à traiter}

La complexité du système et la multiplication des outils ont créé une situation de saturation psychologique et cognitive chez les managers. Plusieurs raisons expliquent cette situation.

Tout d'abord, le nombre important des critères d'amélioration du référentiel ${ }^{6}$ empêche un suivi efficace et interactif. En effet, les managers se voient obliger de gérer et de manipuler un nombre important de critères dont certains sont considérés comme inutiles et inappropriés, car ne servant pas au pilotage des unités opérationnelles.

\footnotetext{
"Energetix Way est une démarche qui va dans le bon sens, cela part d'un bon sentiment mais on arrive à un tel niveau intellectuel, conceptuel que pour faire le lien entre les actions de terrain et la valorisation, que cela ne concerne plus que quelques personnes, même un directeur d'usine finit par être largué. » Directeur financier de la BU
} 
Ensuite, une des spécificités des dimensions de la RSE, réside dans la difficulté de construire des objectifs clairs, précis et chiffrés. Pour contrecarrer ces difficultés, une tendance à multiplier les indicateurs de performance, afin de cerner des aspects complexes, existe au sein du groupe. Mais, ce mouvement a alourdi le système, et ces outils n'ont fait qu'ajouter de nouvelles directives et tâches à des managers qui en avaient déjà en grand nombre. Cela a engendré une apathie progressive des opérationnels considérant ces nouveaux outils plus comme un «fardeau corporate ». Cela ne veut pas dire qu'ils rejettent ou encore qu'ils ne sont pas convaincus par les principes de la RSE. Mais la lourdeur des tâches administratives liées à ces aspects suscite parfois le rejet.
"La déclinaison est soit une désagrégation, soit un changement de métrique (ex de la productivité qui se décline avec des projets), soit parfois un peu artificiel sans vraiment de cohérence. ( ...) Au niveau des établissements, les objectifs se multiplient car déclinaison des objectifs de la BU mais aussi ajout d'autres objectifs propres à chaque établissement». Responsable de la démarche Energetix Way chez la BU

Par ailleurs, au cours des réunions auxquelles nous avons assisté, la revue des indicateurs de reporting et de pilotage se fait d'une manière simultanée et non distincte. En effet, on passe fréquemment de l'examen d'un document de pilotage, propre à la BU à un document de reporting. Ce qui crée des problèmes de compréhension et de suivi, au cours des réunions, de la part de tous les membres du Codir avec des questions du genre : «on est où là ?». Cette confusion est d'autant plus grande que certains indicateurs se ressemblent et se confondent alors qu'ils ont des fonctions et des destinations différentes (certains pour le pilotage issus des budgets, d'autres pour le reporting).
"On a deux outils où on fait deux saisies mais ce sont les mêmes indicateurs. Le fait d'avoir à le mettre dans deux systèmes fait qu'il perd son sens. On ne pilote plus. On fait du reporting. (...)
Il y a sans doute un excès de reporting. Il faut qu'ils établissent un lien avec les établissements. On en transmet plus d'une centaine avec des formats compliqués. Le temps passé est trop important donc forcément quelque chose ne va pas » Directeur d'établissement

De plus, les définitions de certains indicateurs semblent ambiguës. Au fur et à mesure de l'avancée des réunions, nous constatons qu'une certaine forme de lassitude s'installe parmi les membres du Codir et très peu d'entre eux suivent réellement le cours des débats. L'absence d'une priorisation et d'une hiérarchie de ces indicateurs peut expliquer en partie ce phénomène.
"Ce qui est devenu très lourd c'est le reporting, ce n'est pas une activité proportionnelle à l'effectif, donc pour les petits établissements c'est plus lourd. Pour les autoévaluations, vous avez autant de lignes à remplir ici qu'à la plus grande usine du groupe. Il y a une meilleure compréhension des managers des grosses unités que des petites. Il faut alors s'expliquer avec le siège sur les moyens disponibles.» Directeur Sûreté, Sécurité, Santé au Travail, Environnement de la BU.

De plus, dans le référentiel Energetix Way, tous les objectifs ont la même importance.
"Tous les indicateurs sont sur le même plan mais pas en lien avec la réalité. On va y consacrer une énergie qui manquera ailleurs afin de gérer le progrès continu » Directeur Sûreté, Sécurité, Santé au Travail, Environnement de la BU.

Enfin, chaque BU doit se situer par rapport à la politique du groupe. Il peut donc y avoir un conflit entre l'autoévaluation issue du terrain et les besoins globaux exprimés par le corporate. De ce fait, les managers ignorent en partie sur quels aspects ils doivent porter leur attention. Ce qui rend l'utilisation interactive d'Energetix Way difficilement réalisable.

"Le Codir des établissements a été très impliqué mais c'est resté trop codir, la pénétration au sein
des entités est trop hétérogène. Les objectifs de la BU ne sont pas assez diffusés auprès du
personnel. La BU a 3 objectifs stratégiques qui se déclinent en 12 objectifs opérationnels puis 27- 
28 objectifs d'établissement. Le lien entre ces différents éléments est parfois un peu acrobatique.

Certaines unités vont avoir des objectifs complémentaires avec des points communs à atteindre

mais pas assez de dialogue. » Responsable de la démarche Energetix Way chez la BU.

\section{L'influence de la RSE sur le design des systèmes de contrôle}

A l'issue de la description du système de contrôle de la RSE chez Energetix, plusieurs enseignements empiriques et théoriques peuvent être tirés de cette étude de cas.

\subsection{Les enseignements empiriques pour le contrôle de la RSE}

L'objectif de cette recherche est de comprendre les modalités d'utilisation des systèmes de contrôle utilisés pour piloter la performance fondée sur la RSE. Le cas illustre tout d'abord à quoi un tel système de contrôle peut ressembler. Il s'agit d'un cas intéressant dans la mesure où les mécanismes de contrôle de la RSE sont intégrés à part entière avec les autres dimensions de la performance plus traditionnelles contrairement à d'autres systèmes où ils fonctionnent en parallèle (dans les systèmes HSE - Hygiène, Sécurité, Environnement - par exemple).

L'analyse du cas Energetix montre qu'un choix pour un système de contrôle interactif représente la première réponse en termes de design. En effet, ne sachant pas forcément où porter les efforts, la direction du groupe a opté pour un système qui favorise le dialogue continu et fréquent, le partage d'expériences, la discussion, le développement des connaissances et les compétences autour des dimensions de la RSE, etc. Au final, ce système devait permettre de faire émerger de nouvelles stratégies RSE, ainsi que de nouveaux outils pour les décliner.

Néanmoins, l'opérationnalisation de ce système s'est avérée être très délicate. Trop lourd, trop compliqué, trop d'indicateurs, pas assez synthétique sont les principales critiques évoquées par les responsables à l'encontre du système de contrôle RSE, notamment au niveau des unités opérationnelles. L'attention managériale a été saturée, provoquant une surcharge cognitive.

Il devient alors possible d'utiliser cette étude de cas pour examiner un problème théorique plus général lié aux effets d'une surcharge cognitive sur le design des systèmes de contrôle. Dit autrement, cela nous permet de comprendre comment les managers tentent de s'accommoder de systèmes trop complexes. Toutefois, la RSE ayant du mal à être un élément du contrôle interactif à part entière, elle risque ne plus faire partie des préoccupations régulières des managers. Cela a des conséquences sur la perception que ces derniers vont avoir des enjeux de la RSE qui pourraient alors paraitre lointains et déconnectés des autres préoccupations majeures. D'où aussi sans doute, l'impression dans certaines études que la RSE n'est pas un enjeu d'importance pour les managers, ce qui accréditerait alors la thèse d'un découplage entre des préoccupations affichées et des actions effectives. Cela pourrait alors être dû aux spécificités particulières de la gestion de cette performance multicritère.

L'attention managériale au niveau des BU a glissé petit à petit vers d'autres outils de contrôle (des indicateurs de reporting) et ne s'est plus intéressée au système de contrôle de la RSE que par exception. Le schéma suivant résume l'évolution de l'articulation contrôle interactif / contrôle diagnostique. 


\section{Utilisation Interactive}

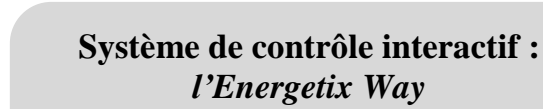

Pour encourager l'apprentissage,

favoriser le dialogue et faire

adopter le changement stratégique

vers des ohiectifs RSF

\section{Utilisation Diagnostique}

12 Indicateurs pour la direction générale

Indicateurs faisant partie du système de reporting vers la hiérarchie et devant être gérés par excention de manière diaonostioue

Utilisation Diagnostique de l'Energetix Way

Surcharge cognitive

Importance de ces indicateurs pour la direction générale

Indicateurs communs à plusieurs niveaux hiérarchioues

Glissement dans l'utilisation avec le temps

\subsection{Les implications théoriques}

L'analyse du système de contrôle d'Energetix montre que les thématiques de la RSE influencent les configurations et l'articulation des systèmes de contrôle. Ces influences se matérialisent dans le choix initial d'une utilisation interactive des outils de contrôle, mais également dans les conséquences managériales que peuvent avoir ultérieurement les thématiques de la RSE sur ces pratiques. Ce constat nous conduit à proposer quatre types d'apports théoriques.

Le premier apport concerne le potentiel de la RSE comme outil interactif. Nous savons que l'utilisation interactive d'un système de contrôle consomme un temps considérable et augmente le nombre et la durée des réunions nécessaires à la vie du système (Tuomela, 2005). Avec la multiplication des indicateurs RSE, la surcharge cognitive a été dépassée, ce qui a des conséquences sur les systèmes de contrôle. Les recherches en psychologie révèlent qu'un problème de surcharge cognitive a des implications sur l'analyse des informations ${ }^{7}$. Neumann et al. (2008) expliquent que les systèmes d'évaluation des performances complexes ${ }^{8}$, s'inscrivant dans une démarche holistique, font l'objet de limitations inconscientes des analyses liées au nombre d'informations analysées.

Proposition 1: La RSE, en multipliant les informations et donc en créant une surcharge cognitive, rend délicate une utilisation interactive des systèmes de contrôle, ainsi que l'implication des managers dans ces systèmes.

Le deuxième apport découle du premier et concerne les interrelations entre les leviers de contrôle interactifs et diagnostique. Le glissement d'Energetix Way d'une utilisation interactive vers une utilisation diagnostique trouve ses origines dans cette surcharge cognitive. Mais cette dernière a provoqué un autre mouvement dans l'articulation et l'utilisation des outils de contrôle. En effet, par manque de temps, les responsables de la BU étudiée ont dû revenir vers une utilisation diagnostique de 
la plupart des outils de contrôle car le système de contrôle s'est retrouvé enliser dans la multiplication des outils qu'il a créés au fil des années (multiplications des indicateurs, des objectifs, etc.). En conséquence, toute l'attention managériale sur la RSE s'est reportée vers un ensemble restreint de 12 indicateurs qui était le plus visible, et le plus critique, pour la direction générale. Toute l'interactivité managériale s'est ainsi transposée d'Energetix Way vers ces 12 indicateurs groupes.

Cela appelle tout d'abord des réflexions sur les interrelations entre levier de contrôle interactif et levier de contrôle diagnostique (Simons, 1994 ; Collier, 2005 ; Widener, 2007). Ces interrelations semblent dépendre, dans le cas observé, de la conception de chacun des deux systèmes. Une forme de substituabilité du contrôle diagnostique au contrôle interactif s'est développée dès lors que le second est devenu trop lourd à manipuler pour les managers. Autrement dit, certains éléments du contrôle interactif sont devenus de fait, malgré les vœux de leurs concepteurs, des outils diagnostiques. Ce mouvement s'est effectué même si le système de reporting diagnostique ne convenait pas aux besoins des managers. Ceux-ci ne sont donc pas libres de choisir l'outil qu'ils souhaitent pour piloter leur performance mais la nature de l'outil les contraint dans leur choix. Simons (1994) a décrit les changements de systèmes interactifs de ou vers des systèmes diagnostiques. Mais il s'agissait alors de mouvements expliqués par des changements de stratégie et des changements de direction générale. Dans le cas présent, on observe un autre phénomène. Le glissement s'opère de l'un à l'autre du fait de la conception à la base du système interactif ${ }^{9}$ et n'est donc pas un phénomène maîtrisé.

Ces conclusions rejoignent les idées d'Abernathy et Chua (1996), pour qui les systèmes de contrôle dans une entreprise se complètent et se substituent en fonction de facteurs de contingences techniques et institutionnelles. Dans le cas d'Energetix, ce sont les spécificités des dimensions de la RSE, ainsi que la surcharge cognitive qui s'en est suivie, qui ont fortement modifié la combinaison des instruments et outils de contrôle (control mix) du groupe.

Proposition 2: Afin de pallier la surcharge cognitive, les systèmes de contrôle diagnostiques se sont substitués aux systèmes interactifs.

Le troisième apport concerne le recours à des indicateurs non financiers dans les systèmes de contrôle devant faciliter la focalisation des managers sur les dimensions clé (Epstein et Manzoni, 1997, Ferreira et Otley, 2009). Mais on voit qu'il n'en ait rien dans le cas étudié. Au contraire, la RSE utilise un grand nombre d'indicateurs non financiers entre lesquels les managers semblent avoir du mal à remettre de la cohérence, et qui ajoute, in fine, de l'opacité et de l'ambigüité au système. Une mise en cohérence et une focalisation est donc à construire de manière extérieure aux informations utilisées, quelles soient financières ou non financières. Des outils comme le Balanced scorecard (Zingales et Hockerts, 2003, Bieker, 2002) peuvent aider les managers à y parvenir mais, comme le montre le design de contrôle mis en œuvre par Energetix, il n'est pas assuré que les managers arrivent pour autant à produire un système de contrôle interactif maîtrisable. Un découplage est alors sans doute nécessaire, où des lieux spécifiques (différents niveaux hiérarchiques par exemple) et des périodes spécifiques d'examen interactif de l'information sont nécessaires. Cela introduit une division $\mathrm{du}$ travail temporelle et spatiale qui semble consubstantielle à l'utilisation interactive d'un système de contrôle. L'interactivité est donc un problème organisationnel complexe dont l'étude nécessite la prise en compte de l'articulation des différents dispositifs de contrôle (le control mix selon Collier, 2005), ceux-ci étant activés à différents niveaux de l'entreprise selon des périodicités différentes. L'interactivité est donc davantage une succession de couches de systèmes interactifs en interaction les uns avec les autres qu'un phénomène localisé, par exemple sur les top managers pour reprendre Simons. 
Proposition 3: Afin de pallier la surcharge cognitive, le système de contrôle de la RSE peut être découplé hiérarchiquement afin de cantonner l'analyse interactive de certaines informations de la RSE à des niveaux hiérarchiques donnés.

Le quatrième apport concerne le type d'indicateurs choisis pour promouvoir l'interactivité. Lipe and Salterio $(2000)^{10}$ ont montré que, dans l'évaluation comparative de la performance des managers, la surcharge cognitive conduisait les évaluateurs à privilégier un nombre plus réduit d'indicateurs. En l'occurrence, les managers semblent privilégier les indicateurs qu'ils ont en commun avec d'autres niveaux hiérarchiques. C'est bien précisément ce qu'ont fait les managers d'Energetix, en se repliant sur les données du reporting. Celles-ci étaient critiquées car pas toujours pertinentes pour des enjeux locaux de gestion mais elles avaient en revanche l'avantage d'être peu nombreuses et d'intéresser la direction générale. Le troisième apport montrait que les indicateurs interactifs font l'objet d'une spécialisation temporelle et spatiale qui permet de traiter les enjeux au plus près de l'endroit où les questions se posent. Notre quatrième apport, partiellement contradictoire avec le troisième, montre que l'interactivité est finalement plus complexe, puisque des indicateurs communs sont aussi des candidats à une discussion interactive entre les niveaux hiérarchiques. Cela implique donc qu'il existe une interactivité interne à un lieu donné et une interactivité faisant le lien entre deux niveaux hiérarchiques donnés.

Proposition 4: Afin de pallier la surcharge cognitive, les managers privilégient l'analyse interactive d'indicateurs communs avec d'autres niveaux hiérarchiques.

\section{Conclusion}

Deux principales conclusions peuvent être soulevées à l'issue de cette recherche. Tout d'abord, cet article contribue empiriquement aux recherches portant sur la conception des systèmes de contrôle de la RSE. Les résultats de cette recherche posent la problématique de l'intégration des dimensions de la RSE au pilotage de la performance organisationnelle. A ce titre, l'étude du cas Energetix apporte un éclairage sur l'une des rares tentatives d'intégration des trois axes de la RSE dans un seul et unique système managérial au sein des groupes français. Néanmoins, ce cas illustre toutes les difficultés de ces pratiques, au premier plan desquelles, le manque de temps nécessaire pour le pilotage des nouvelles dimensions de la RSE.

D'un point de vue théorique, les contributions de cette recherche mettent au jour les effets que peuvent impliquer ces phénomènes de surcharges cognitives sur les systèmes de contrôle des entreprises: glissement d'interactif vers diagnostique et inversement, priorisation des outils de contrôle, découplage et spécialisation hiérarchique, modification du control mix, etc.

La RSE est un nouvel enjeu pour les organisations. Au-delà de la nécessité de prendre en compte des dimensions environnementales et sociales, la RSE suppose surtout leur intégration dans les systèmes managériaux, au même titre que la dimension financière et économique. Mais ceci pose un véritable défi pour les managers car cette intégration bouleverse des systèmes déjà difficilement maîtrisables avec la seule dimension financière. 


\section{Notes}

1. On peut définir la Responsabilité Sociétale de l'Entreprise comme « Les modalités de réponse de l'entreprise aux interpellations sociétales en produisant des stratégies, des dispositifs de management, de conduite de changement et des méthodes de pilotage, de contrôle, d'évaluation et de reddition» (Capron et Quairel-Lanoizelée, 2007)

2. Les systèmes de croyances et de contraintes existent dans le cas étudié. Toutefois, par manque de place et parce qu'ils ne sont pas centraux dans notre analyse, nous avons délibérément choisi de ne pas les développer.

3. Cette recherche s'intéresse particulièrement à l'étude des pratiques relevant de la RSE. Le terme «développement durable», qui pour nous a un sens plus macro-économique et moins managériale que la RSE, n'est utilisé dans cet article que par référence au langage interne à l'entreprise étudiée, dans laquelle on parle de «démarche développement durable » ou encore de "direction du développement durable et du progrès continu ».

4. Ainsi une partie des objectifs a été modifiée d'un exercice à l'autre car certains objectifs de n-1 avaient été atteints. Même en cours

\section{Bibliographie}

Abernathy, M.A., Chua,W.F. (1996). A field study of control system "redesign": the impact of institutional processes on strategic choice. Contemporary Accounting Research, 13: 569581.

Abernethy, M.A., Brownell, P. (1999). The role of budgets in organizations facing strategic change: an exploratory study. Accounting, Organizations and Society. 24(3), pp.189-204.

Berland, N., Loison, M.C. (2008). Responsible care and sustainable management: the double influence of society - management practices. Society \& Business Review 3(1): 41-56.

Bieker, T. (2002). Managing corporate sustainability with the Balanced Scorecard: Developing a Balanced Scorecard for Integrity Management. Oikos PhD summer academy.

Bisbe, J., Otley. D. (2004). The effects of the interactive use of management control systems d'année, certains objectifs sont relégués au second plan dès lors que les managers considèrent qu'ils ne posent plus d'enjeux particuliers.

5. Un exemple de ce découplage, entre les items traités au niveau de la BU et les items traités au niveau d'un des établissements, est fourni en annexe 3.

6. Il existe une centaine de critères d'amélioration que doit suivre chaque BU.

7. Halford et al. (2005) ont montré par exemple qu'un être humain ne pouvait mentalement analyser plus de quatre variables simultanément au risque d'affaiblir la qualité de son jugement.

8. Tel que le Balanced Scorecard proposant généralement entre seize et vingt quatre indicateurs.

9. Dans le cas présent, c'est la conception du système interactif qui entraîne un glissement. On pourrait très bien imaginer qu'il soit possible théoriquement que ce glissement soit initié par la conception du système diagnostique. Mais notre cas ne nous montre que le premier type de phénomène.

10. Utilisant notamment les travaux des psychologues Slovic et MacPhillamy (1974).

on product innovation. Accounting, Organizations and Society 29(8): 709-737.

Bouquin, H. (2008). Le contrôle de gestion. PUF: $8^{\text {ème }}$ édition.

Bruining, H., Bonnet, M., Wright, M. (2004). Management control systems and strategy change in buyouts. Management Accounting Research 15(2): 155-177.

Burnett, R., Hansen, D. (2008). Eco-Efficiency: A Role for Environmental Cost Management. Accounting, Organizations, and Society 33 (6): 551-581.

Capron, M., Quairel-Lanoizelée, F. (2004). Mythes et Réalités de l'Entreprise Responsable. Paris: La Découverte.

Capron, M., Quairel-Lanoizelée, F. (2006). Evaluer les stratégies de développement durable des entreprises : l'utopie mobilisatrice de la performance globale. Revue de l'Organisation Responsable 1 : 5-17. 
Capron, M., Quairel-Lanoizelée, F. (2007). La responsabilité sociale d'entreprise. Paris : La Découverte.

Collier, P. M. (2005). Entrepreneurial control and the construction of a relevant accounting. Management Accounting Research 16(3): 321339.

Damak Ayadi, S. (2006). Analyse des facteurs explicatifs de la publication des rapports sociétaux en France. Comptabilité - Contrôle Audit 12 (2) :93-116.

Dambrin, C., Löning, H. (2008). Systèmes de contrôle interactifs et théories de l'apprentissage : une relecture des travaux de R. Simons à l'aune des théories piagétiennes. Comptabilité - Contrôle - Audit, numéro spécial «Comptabilités et Connaissances » : 113-140.

Epstein, M.J., Manzoni, J.F. (1997). The Balanced Scorecard and Tableau de Bord: translating strategy into action. Management Accounting (USA) 79 (2): 28-37.

Ferreira, A., Otley, D. (2009). The design and use of performance management systems: An extended framework for analysis. Management Accounting Research 20 (4): 263-282.

Germain, C., Gates, S. (2007). Le niveau de développement des indicateurs de responsabilité sociale dans les outils de pilotage de contrôle de gestion: une analyse des pratiques des entreprises. $28^{\text {ème }}$ congrès annuel de l'Association Francophone de Comptabilité, Poitiers.

Halford, G. S., R. Baker, J. E. McGredden, et J. D. Bain. (2005). How many variables can humans process? Psychological Science 16(1): 70-76.

Henri, J. F., Journeault, M. (2010). Eco-control: The influence of management control systems on environmental and economic performance. Accounting, Organizations and Society 35 (1): 63-80.

Hockerts, K., (2001). Corporate Sustainability Management, Towards Controlling Corporate Ecological and Social Sustainability. in Proceedings of Greening of Industry Network Conference, January 21-24, Bangkok.

IFAC, International Federation of Accountants (2005), International Guidelines Document on Environmental Management Accounting (EMA).

Ittner, C.D., Lacker, D.F. (2004). Coming Up Short on Nonfinancial Performance Measurement. Harvard Business Review Nov: 88-93.
Langfield-Smith, K. (1997). Management control systems and strategy: a critical review. Accounting, Organizations and Society 2(2): 207-232.

Lingle, J.H., Schiemann, W.A., (1996). From balanced scorecard to strategic gauges: Is measurement worth it? Management Review 85: 56-61.

Lipe, M., Salterio, S. (2000). The balanced scorecard: Judgmental effects of common and unique performance measures. The Accounting Review 75 (3): 283-298.

Loison, M.C., Pezet, A., (2006). L'entreprise verte et les boues rouges: Les pratiques controversées de la responsabilité sociétale à l'usine d'alumine de Gardanne (1960-1966). Entreprises et Histoire 45.

Marginson, D.E.W. (2002). Management control systems and their effects on strategy formation at middle-management levels: evidence from a U.K. organization. Strategic Management Journal 23: 1019-1031.

Marquet-Pondeville S. (2003). Le contrôle de gestion environnemental: une approche théorique contingente et une étude empirique du cas des entreprises manufacturières belges. Presses Universitaires de Louvain - UCL.

Melnyk, S.A., Sroufe, R.P., Calantone, R. (2003). Assessing the impact of environmental management systems on corporate and environmental performance. Journal of Operations Management 21: 329-351.

Meyssonnier F. et F.Rasolofo-Distler (2008), « le contrôle de gestion entre responsabilité globale et performance économique : le cas d'une entreprise sociale pour l'habitat », Comptabilité - Contrôle - Audit, Tome 14 - Volume 2 Décembre.

Miles, M.B., Huberman, A.M. (2003). Analyse des données qualitatives. $2^{\text {ème }}$ Edition, De Boeck.

Mocquet, A.C., Pezet, A. (2006). Les technologies de la responsabilité sociétale ou l'invention du manager responsable. Finance, Contrôle, Stratégie 9 (4) : 113-142

Moquet A.C. (2008). Les systèmes de contrôle d'une stratégie de responsabilité sociétale. Les cas Lafarge et Danone. Doctorat en sciences de gestion, Paris: Université Paris Dauphine.

Neumann, B.R., Roberts, M. L., Cauvin, E. (2008). Financial and nonfinancial performance measures. Cost Management 22 (6): 5-14. 
Orlitzky, M., Schmidt, F.L, Rynes, S. (2003). Corporate Social and Financial Performance: A Meta-analysis. Organization Studies 24(3): 403441.

Osborn, C.S. (1998). Systems for sustainable organizations: emergent strategies, interactive control and semi-formal information. Journal of Management Studies 35 (4) : 481-509.

Oxibar, B. (2003). La diffusion d'informations sociétales dans les rapports annuels et les sites Internet par les entreprises françaises, Thèse en Sciences de Gestion, Université de Paris 9 Dauphine, Paris.

Pizzini, M. (2006). The relation between costsystem design, managers' evaluations of the relevance and usefulness of cost data and financial performance: An empirical study of US hospitals. Accounting, Organizations and Society 31 (2): 179-210.

Preston, L., O’Bannon, D. (1997). The corporate social-financial performance relationship. Business and Society 36(4): 419-429.

Quairel, F. (2006). Contrôle de la performance globale et RSE. $27^{\text {ème }}$ congrès annuel de l'Association Francophone de Comptabilité, Tunis.

Richard, P.J., Devinney, T.M., Yip, G.S., Johnson G. (2009). Measuring organizational performance: Towards methodological best practice. Journal of management 35(3): 718804.

Schick, A. G., Gordon, L. A., Haka, S. (1990). Information Overload: a Temporal Approach. Accounting, Organisations and Society 17(8): 199-220.

Simons, R. (1987a). Accounting control systems and business strategy: an empirical analysis. Accounting, Organizations and Society 12 (4): 357-374.

Simons, R. (1987b). Planning, Control, and Uncertainty: A Process View. in W.J. Bruns et R.S. Kaplan (dir.), Accounting \& Management.
Field Study Perspectives: 338-362. Harvard Business School Press

Simons, R. (1990). The Role of Management Control Systems in Creating Competitive Advantage: New Perspectives. Accounting, Organizations and Society 15(1-2): 127-143.

Simons, R. (1991). Strategic orientation and top management attention to control systems. Strategic Management Journal, 12: 49-62.

Simons, R. (1994). How new top managers use control systems as levers of strategic renewal. Strategic Management Journal 15: 169-189.

Simons, R. (1995). Levers of Control. Harvard University Press, Boston.

Slovic, P., MacPhillamy, D. (1974). Dimensional commensurability and cue utilization in comparative judgment. Organizational Behaviour and Human Performance 11: 172194.

Tesch, R. (1990). Qualitative research: Analysis types and software tools. New York: Falmer Press.

Tuomela, T. S., (2005). The interplay of different levers of control: A case study of introducing a new performance measurement system. Management Accounting Research 16(3): 293 320.

Vaivio, J. (1999). Exploring a Non-Financial Management Accounting Change. Management Accounting Research. 10(4): 409-437.

Vaivio, J., (2001). Non-Financial Measurement in an Organizational Context: Three Perspectives. Ph.D. Thesis, Helsinki School of Economics and Business Administration

Widener, S. K. (2007). An empirical analysis of the levers of control framework. Accounting, Organizations and Society 32(7-8): 757-788.

Zingales, F. Hockerts, K. (2003). Balanced Scorecard and Sustainability: Examples from Literature and Practice. INSEAD working paper 30, CMER, Fontainebleau, France. 


\section{Annexe 1 : Responsables rencontrés chez le groupe Energetix}

\begin{tabular}{|l|l|}
\hline \multicolumn{1}{|c|}{ Fonction } & \multicolumn{1}{c|}{ Niveau hiérarchique } \\
\hline Directeur du développement durable et du progrès continu & Groupe \\
\hline Vice-président Qualité et progrès continu & Groupe \\
\hline Directeur de la Business Unit Chimie & BU Chimie \\
\hline Leader progrès continu dans la BU Chimie & BU Chimie \\
\hline Direction communication & BU Chimie \\
\hline Direction industrielle & BU Chimie \\
\hline $\begin{array}{l}\text { Responsable des investissements - directeur de la maîtrise } \\
\text { d'ouvrage }\end{array}$ & BU Chimie \\
\hline Directeur financier & BU Chimie \\
\hline Directeur Sûreté, Sécurité, Santé au Travail, Environnement & BU Chimie \\
\hline Délégué industrialisation & BU Chimie \\
\hline Directeur d'établissement & Usine 1 appartenant à la BU Chimie \\
\hline Directeur d'établissement & Usine 2 appartenant à la BU Chimie \\
\hline Directeur d'établissement & Usine 3 appartenant à la BU Chimie \\
\hline Directeur d'établissement & Usine 4 appartenant à la BU Chimie \\
\hline
\end{tabular}

\section{Annexe 2: Les dix engagements stratégiques d'Energetix et le processus d'autoévaluation}

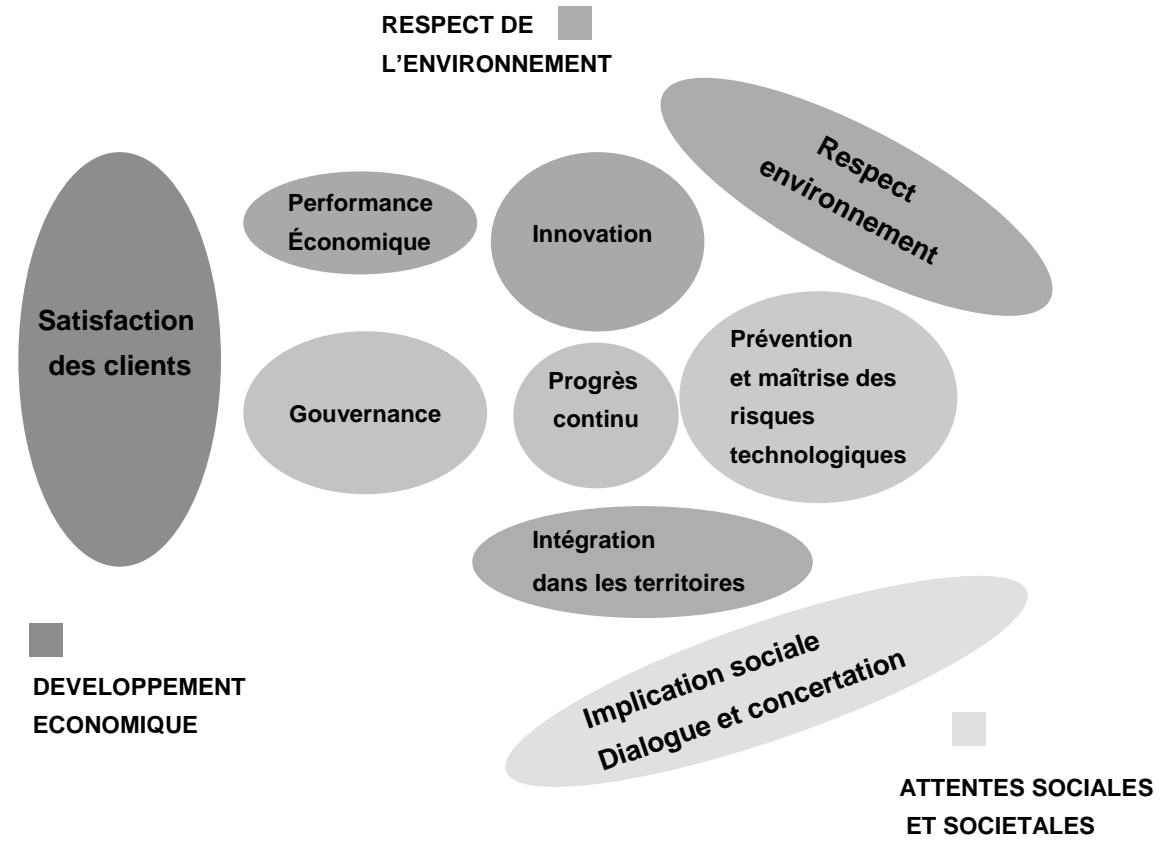



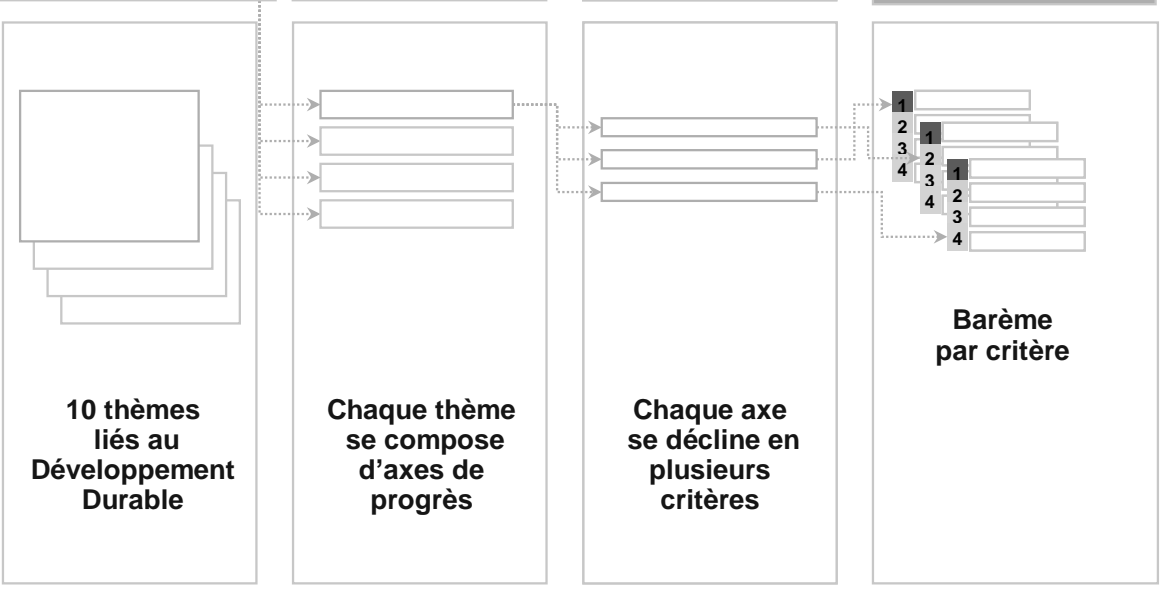

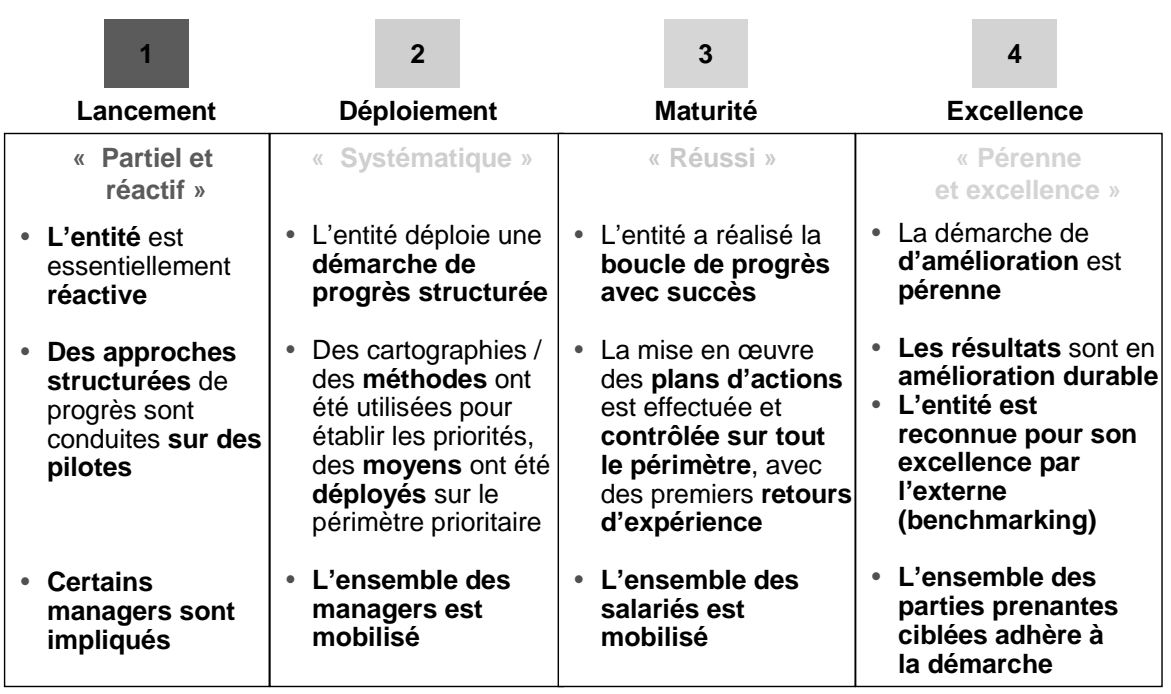

Thème 9 : Dialogue et concertation Axe 91. Relations avec les parties prenantes

\begin{tabular}{|l|l|}
\hline \multicolumn{1}{|c|}{} & 912. Organisation du dialogue avec les parties prenantes \\
\hline 1 & $\begin{array}{l}\text { Les relations de l'entité avec des parties prenantes prioritaires / ciblées se limitent aux } \\
\text { structures imposées (Commission Locale d'Information,...) ou sont très occasionnelles. }\end{array}$ \\
\hline 2 & $\begin{array}{l}\text { L'entité participe de manière systématique aux structures existantes lui permettant } \\
\text { d'échanger avec des parties prenantes ciblées. }\end{array}$ \\
\hline 3 & $\begin{array}{l}\text { L'entité a développé, en cohérence avec l'approche du Groupe, les structures } \\
\text { spécifiques qui lui paraissent nécessaires au dialogue avec certaines parties prenantes } \\
\text { ciblées. } \\
\text { Elle s'est assurée qu'elle atteint ainsi toutes les parties prenantes ciblées avec le niveau } \\
\text { d'approche pertinent (local, régional, national, international,...). }\end{array}$ \\
\hline 4 & $\begin{array}{l}\text { L'entité s'assure régulièrement auprès des parties prenantes ciblées qu'elles sont } \\
\text { satisfaites de l'organisation du dialogue. }\end{array}$ \\
\hline $\begin{array}{l}\text { Les structures de communication sont adaptées en fonction des réponses et de } \\
\text { l'évolution du panel de parties prenantes ciblées. }\end{array}$ \\
\hline
\end{tabular}




\section{Annexe 3 : Exemple de découplage dans le traitement des critères Energetix Way}

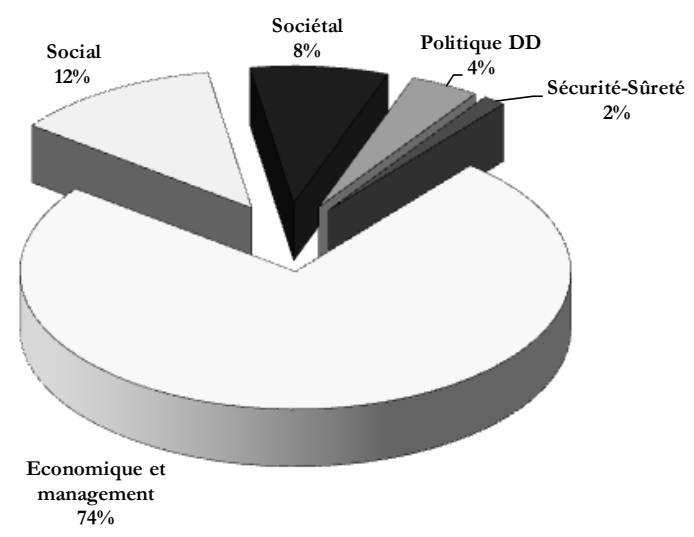

Répartition des items Energetix Way traités au niveau de la BU en 2006

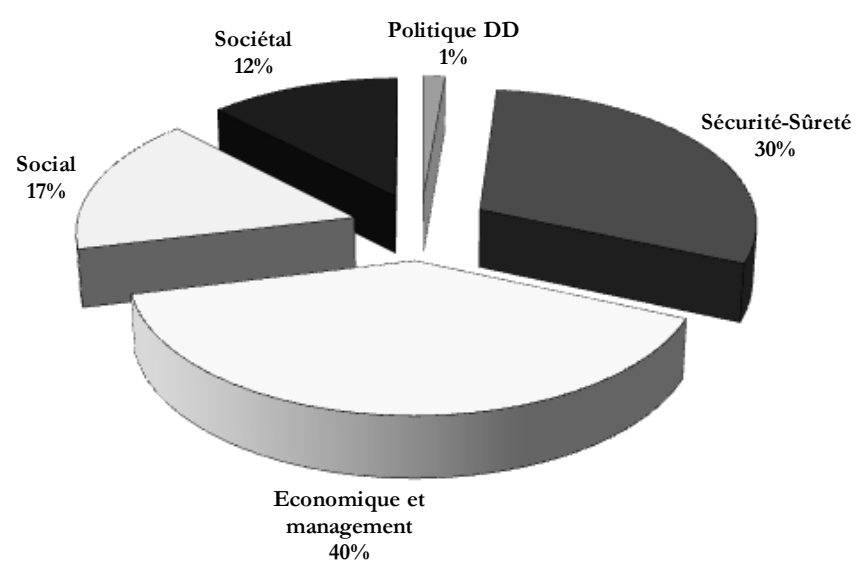

Répartition des items Energetix Way traités au niveau d'un établissement industriel en 2006

Parmi les indicateurs qui ne sont plus traités au sein de la BU, mais plutôt au sein des unités industrielles, on trouve les indicateurs relevant des thèmes 5 et 6 des 10 engagements « développement durable » du groupe.

Les figurent ci-dessous fournissent un exemple des indicateurs prévues pour le critère 5 , qui ne donnent plus forcément lieu à des discussions au niveau de la BU. 
Thème 5: Prévention et maîtrise des risques technologiques

Les critères

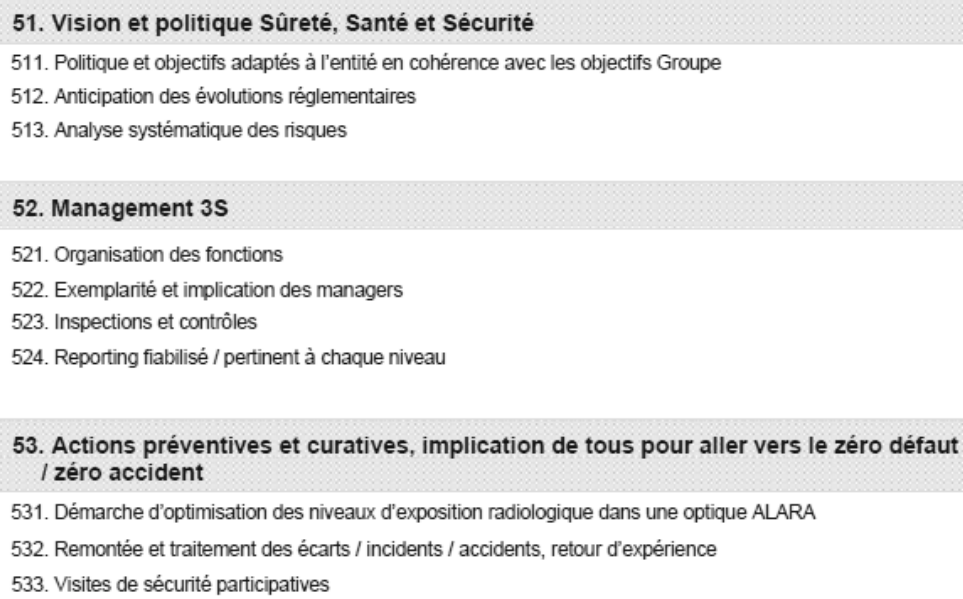

Thème 5: Prévention et maîtrise des risques technologiques Axe 51. Vision et politique Sûreté, Santé et Sécurité

\begin{tabular}{|l|l|}
\hline \multicolumn{1}{|c|}{ Indicateurs } & Description / commentaires \\
\hline $\begin{array}{l}\text { - Nombre de réunions organisées pour présenter } \\
\text { les objectifs } 3 \mathrm{~S}\end{array}$ \\
\begin{tabular}{|l|l|}
\hline \% de salariés auxquels les objectifs $3 S$ ont été \\
présentés
\end{tabular} \\
\hline $\begin{array}{l}\text { - Nombre de non-conformités nouvelles (créées } \\
\text { par une évolution de la réglementation) }\end{array}$ \\
\hline
\end{tabular}

\section{Thème 5: Prévention et maîtrise des risques technologiques} Axe 52. Management 3S

\begin{tabular}{|c|c|}
\hline Indicateurs & Description / commentaires \\
\hline $\begin{array}{l}\text { Nombre de formations dans les domaines } 3 S \\
\text { prévues et taux de réalisation }\end{array}$ & $\cdot-$ \\
\hline $\begin{array}{l}\text { \% de salariés et des personnels travaillant sur } \\
\text { le site ayant participé à une formation } 3 \mathrm{~S}\end{array}$ & $\cdot-$ \\
\hline - Nombre d'inspections & $\cdot-$ \\
\hline - \% d'actions correctives soldées & - Par rapport au nombre d'actions correctives totales \\
\hline $\begin{array}{l}\text { - Nombre d'inspecteurs internes assurant des } \\
\text { inspections à l'extérieur de l'entité }\end{array}$ & $\cdot-$ \\
\hline $\begin{array}{l}\text { - Nombre d'indicateurs 3S suivis (dont suivis par } \\
\text { le Groupe, dont suivis par l'entité) }\end{array}$ & - - \\
\hline
\end{tabular}




\section{Annexe 4: Exemples des indicateurs «groupe» destinés à la direction générale}

\begin{tabular}{|c|c|c|c|c|c|c|}
\hline SECURITE & 2003 & 2004 & 2005 & 2005 & 2006 & 2007 \\
\hline Accidents du travail, avec arrêts & réel & réel & objectif & réel & objectif & objectif \\
\hline \multicolumn{7}{|l|}{ Taux de fréquence } \\
\hline \multicolumn{7}{|l|}{ Taux de gravité } \\
\hline DOSIMETRIE & 2003 & 2004 & 2005 & 2005 & 2006 & 2007 \\
\hline Dose moyenne d'exposition aux RI (mSv) & réel & réel & objectif & réel & objectif & objectif \\
\hline \multicolumn{7}{|l|}{ Salariés du Groupe } \\
\hline \multicolumn{7}{|l|}{ Personnel sous-traitant } \\
\hline \multicolumn{7}{|l|}{ Nombre de personnes $>14 \mathrm{mSv}$} \\
\hline \multicolumn{7}{|l|}{ Nombre de personnes $>20 \mathrm{mSv}$} \\
\hline \multirow[t]{2}{*}{ ENVIRONNEMENT } & 2003 & 2004 & 2005 & 2005 & 2006 & 2007 \\
\hline & réel & réel & objectif & réel & objectif & objectif \\
\hline \multicolumn{7}{|l|}{$\begin{array}{l}\text { Eau prélevée, en milliers de m3 } \\
\text { / à un indice d'activité pertinent }\end{array}$} \\
\hline \multicolumn{7}{|l|}{$\begin{array}{c}\text { Energie électrique consommée, en } \\
\text { GWh } \\
\text { / à un indice d'activité pertinent }\end{array}$} \\
\hline \multicolumn{7}{|l|}{$\begin{array}{c}\text { Energie fossile consommée, en GWh } \\
\text { / à un indice d'activité pertinent }\end{array}$} \\
\hline $\begin{array}{c}\text { Papier consommé, en tonnes } \\
\text { en kg par personne }\end{array}$ & & & & & & \\
\hline
\end{tabular}

